\title{
Lower third molar inclusion associated with paraesthesia: A case report
}

\author{
ALESSANDRO ESPEDITO DI LAURO $^{1 *}$, MARIUS BOARIU ${ }^{2}$, PASQUALE SAMMARTINO ${ }^{1}$, \\ FABIO SCOTTO $^{1}$, ROBERTA GASPARRO ${ }^{1}$, STEFAN-IOAN STRATUL ${ }^{3 *}$, DARIAN RUSU ${ }^{3 *}$, \\ ALEXANDRA ROMAN $^{4}$, PETRA SURLIN ${ }^{5}$ and SORINA SOLOMON ${ }^{6}$ \\ ${ }^{1}$ Department of Oral Surgery, University of Naples Federico II, I-80131 Napoli, Italy; Departments of ${ }^{2}$ Endodontics and \\ ${ }^{3}$ Periodontology, Faculty of Dental Medicine, 'Victor Babes' University of Medicine and Pharmacy, 300041 Timisoara; \\ ${ }^{4}$ Department of Periodontology, 'Iuliu Hatieganu' University of Medicine and Pharmacy, 400000 Cluj-Napoca; \\ ${ }^{5}$ Department of Periodontology, University of Craiova, 200349 Craiova; ${ }^{6}$ Department of Periodontology, \\ 'Gr. T. Popa' University of Medicine and Pharmacy, 700020 Iasi, Romania
}

Received March 29, 2021; Accepted April 28, 2021

DOI: $10.3892 / \mathrm{etm} .2021 .10258$

\begin{abstract}
When lower third molar inclusion is associated with neurosensorial complications, the treatment of choice is its surgical avulsion. One of these complications, that may be the most alarming during a first medical examination, is hemi-lip paraesthesia, that can appear in the presence of several mandibular lesions. This is a report of a rare clinical case in which paraesthesia was linked to the closeness between the root block of the dental element and the mandibular canal, which houses the neurovascular trunk of the lower mandibular nerve. A 64 year-old male Caucasian patient, presented with the chief complaint of past periodic inflammatory events in the retromolar region of the oral cavity and hemi-lip paraesthesia. Upon local clinical and radiological examination, a lower left third molar with class 3 position $\mathrm{C}$ inclusion was incriminated. The medical history of the patient revealed well compensated diabetes mellitus type II, and pharmacologically controlled hypertension. The tooth was surgically removed using piezoelectric instruments. Before and after surgery, three types of tests (tactile, pain and thermal sensitivity) were carried out to delimit the area affected by paraesthesia. At 7 days, the area of hypoesthesia of the hemi-lip was significantly reduced. Further improvement in tactile and thermal sensitivity occurred in subsequent follow-up, at 1 and 3 months, postoperatively.
\end{abstract}

Correspondence to: Dr Marius Boariu, Department of Endodontics, Faculty of Dental Medicine, 'Victor Babes' University of Medicine and Pharmacy, 9 Boulevard of 1989 Revolution, 300041 Timisoara, Romania

E-mail: boarium@yahoo.com

${ }^{*}$ Contributed equally

Key words: dental inclusion, third molar, avulsion, dysodontiasis, paraesthesia, piezosurgery
This clinical case demonstrates that the surgical intervention performed with piezoelectric instruments prevented the damage of an important structure such as the lower mandibular nerve, and promoted regression of a contingent paraesthesia.

\section{Introduction}

Third molars have been described as different from other teeth in the oral cavity. They have the highest rate of developmental abnormalities and, most importantly, are last in the eruption sequence (1). Often third molars are subject to anomalies of eruption, such as inclusion, retention, impaction, or inclusion decay, affecting the population in $71 \%$ of cases (2).

One of the causes identified by several authors is the lack of space for the eruption of lower third molar (3).

In 1979, the National Institute of Dental Research of the USA sponsored a Consensus Development Conference about lower third molars avulsion. It included workshops that focused on topics such as the effects of the extraction of third molars on their growth and development, the timing and technical considerations for avulsions, prosthetic and periodontal considerations, postoperative morbidity, and advantages and disadvantages of the extraction of third molars (4). The indications confirmed to date for the avulsion of third molars include the increased frequency of local inflammation, tooth decay or periodontal damage of the second molar, cystic degeneration of the pericoronary (follicular) sac, and neuralgia associated with involvement of the mandibular nerve (5-7).

Third molar extraction is the most frequent procedure in oral surgery. A descriptive study made of 319 patients subjected to surgical removal of a third molar in the context of the Master of Oral Surgery and Implantology of the Barcelona University Dental School, Barcelona, Spain between July 2004 and March 2005 evaluated the following parameters: Sex, age, molar, type of impaction, position according to the classifications of Pell and Gregory and of Winter, and the reasons justifying extraction. The study concluded that prophylaxis was the principal indication of third molar 
extraction, followed by orthodontic reasons. Regarding third molars with associated clinical symptoms or signs, infectious disease, including pericoronitis, was the pathology most often observed by the oral surgeon, followed by caries (8). It is worth to note that in some regions of the world, prophylactic surgical extraction of third molars is not a common practice (1).

A study measuring the prevalence of disease of mandibular third molars referred for removal found pericoronitis in $64 \%$ of cases, with odds ratio about 22 and 34 times higher for molars partially covered by soft tissue than for molars completely covered by soft or bone tissue (9). Another study analyzed the occurrence of symptoms of unerupted mandibular third molars, and investigated associated pathologies, to determine indications for removal of unerupted mandibular third molars in a Turkish population. The study found that $62.6 \%$ of all unerupted third molars had no symptoms, while $37.4 \%$ were associated with symptoms. The most frequent complaints of the patients were pain and swelling, and pericoronitis was observed as the most frequent pathology $(10,11)$. A vertical position predominated among the third molars with associated pathology (8).

Upon investigation of the relevant literature, to the best of the authors' knowledge, no study or case presentation has reported to date paresthesia as a dominant pre-operative symptom of pericoronitis of third mandibular molars. The sole article reported an odontogenic paresthesia was a case report from 1986, that describes selective anesthesia of peripheric branches of the trigeminal nerve, due to an apical lesion on the second right lower molar (12). On the other hand, most of the literature on impacted third molars reports exclusively on postsurgical sensory impairment following extraction $(13,14)$.

\section{Case presentation}

A 64-year old male Caucasian patient presented to the ambulatory services of the Department of Oral Surgery, University of Naples Federico II, Italy, complaining of periodic inflammatory events in the retromolar region of the oral cavity. At anamnesis, the patient reports about well compensated type II diabetes mellitus and pharmacologically controlled hypertension with valsartan/hydrochlorothiazide (Combisartan) 160/12.5 mg, and amlodipine (Norvasc) $5 \mathrm{mg}$, 1 capsule daily. The frequency and the intensity of the main clinical symptoms suggested the disodontiasis of tooth 3.8 but included permanent paraesthesia of the left hemi-lip, which was accentuated during inflammatory episodes. As a result, the quality of life of the patient was severely affected.

Upon clinical and radiological investigation, the following diagnosis was established: Severe, generalized periodontitis stage IV-grade C; Kennedy class II with one modification in the mandible; subtotal maxillary edentation; severe caries on lower left first premolar, lower right second premolar, and first molar. The examination of the panoramic radiograph revealed the inclusion of both third lower molars, both in the horizontal position and in apparent contiguity with the mandibular canal (Fig. 1). On the computed tomography (CT) sections pertaining to the molar's crown, the interruption of the white lines and a compression of the mandibular canal lumen by the impacted molar's crown was visible (Fig. 2). The CT examination confirmed that the lower left third molar had two fused roots, which had relationships of contiguity with the mandibular canal (Fig. 3). According to the classification of Pell and Gregory, the lower left third molar was in class 3 position $C$ (15). To assess the tactile, pain and thermal sensitivity, three types of tests were carried out to delineate and demarcate the area affected by paraesthesia at baseline (Fig. 4).

The aim of the test for tactile sensitivity was conducted to evaluate the large, myelinated fibers $\mathrm{A} \alpha$, for fast and slow adaptation. This sensitivity was evaluated by using nylon sutures, slid in a direction perpendicular to the skin, by asking the blind-folded patient whether the stimulus evoked or not a specific sensation. Evaluation of the thermo-nociceptive sensitivity tested the myelinated fibers $A \delta$ and $C$. The sensation was evoked using cotton pellets soaked in ethyl chloride or a pencil of ice. The pain can be evoked more easily using thin needles (16). These tests were performed on those skin areas that already had been previously outlined by a demographic pencil, as areas in which the patient reported to have alterations in sensitivity; thus drawing an initial mapping.

Because of the patient's medical history of diabetes mellitus type II, antibiotic prophylaxis with amoxicillin $2 \mathrm{~g}$ orally $1 \mathrm{~h}$ before surgery was administrated. The surgical procedure was planned under regional anesthesia, using piezosurgery (Mectron S.p.A.) to improve the intra-operative and post-operative sensitivity and the surgical control in the vicinity of important anatomical structures, such as neurovascular structures.

After rinsing for disinfection of the oral cavity with Betadine (povidone iodine 10\%), a mucoperiosteal flap was created in the retro-molar trigone with release incisions distal to the second premolar. Once the flap was raised and retracted, the projection of the horizontally retained molar on the crestal cortical was established, and an oblongated fenestration of the bone right above the retained tooth was performed, thus highlighting the distal aspect of the crown of the included third molar. Once the fenestration was suitably enlarged, the pericoronary sac became apparent and was partially removed (Fig. 5), leaving the crown exposed.

The coronal odontotomy was performed using the piezoelectric handpiece (insert OT7-Ex1), completing the cut in the vicinity of the lingual cortical (Fig. 6); a root elevator was used to mobilize and to remove the coronal fragment. With the aid of two other root elevators (thin straight and curved), once having established a slight mobility of the remaining intra-osseous fragment, the latter was advanced along the long axis of the root trunk, without the need for further separation, and was removed (Fig. 7). The bone margins of the osseous crypt were regularized (Fig. 8). The bone cavity was irrigated with a combination of antibiotic solution $220 \mathrm{mg}$ ampicillin plus 147 mg sulbactam (Unasyn, Haupt Pharma Latina S.R.L.), $1 \mathrm{~g} / 3.2 \mathrm{ml}$ solution, and prednisone (Deltacortenesol $25 \mathrm{mg}$ vials, Bruno Farmaceutici S.p.A). After hemostasis was achieved, the flap was repositioned and sutured with $3 / 0$ silk sutures (Med-Silk, Med-Europe).

The healing occurred uneventful. After 7 days, the stitches were removed. The area of hypoesthesia of the hemi-lip was significantly reduced even at this early checkup, as evidenced by the thermo-demographic test (baseline). Further improvement of tactile and thermal pain was demonstrated in subsequent follow-up at 1 and 3 months, postoperatively (Fig. 9). 


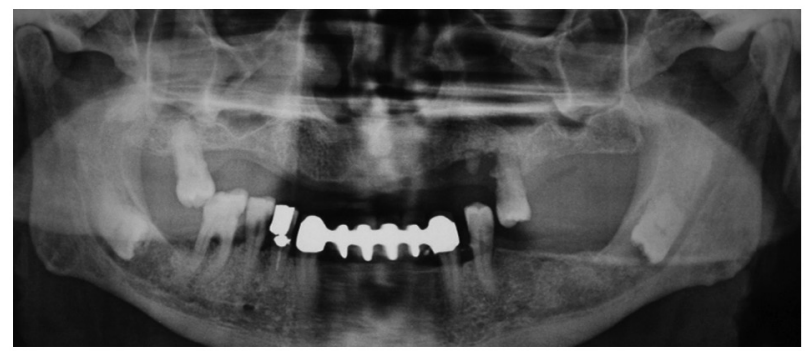

Figure 1. Preoperative panoramic radiograph. Note the slight radiolucency neighboring the apical half of the occlusal aspect, which may signify a developing inflammatory lesion in the vicinity of the mandibular canal.

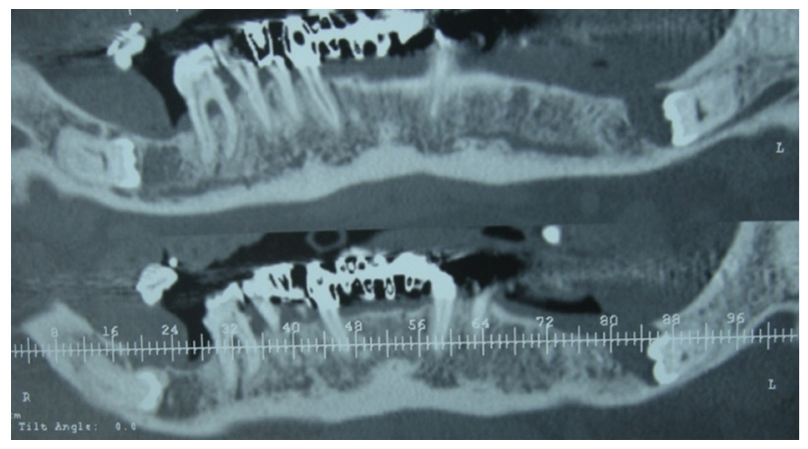

Figure 2. Mid-mandibular computed tomography (CT) section. Note the close vicinity/apparent invasion of the mandibular canal by the mesial aspect of the crown.

\section{Discussion}

An early literature review analyzing the risks and benefits associated with avulsion of third molars included four possible different clinical situations: Risk of non-intervention, risk of intervention, benefit of non-intervention and benefit of intervention (17). According to the authors, the risks in the case of non-intervention are crowded teeth (when supported by the analysis of growth forecasts); resorption of the adjacent tooth and periodontal problems; development of infections, cysts, and tumors.

The risks in the case of intervention are minor transient (paraesthesia, alveolitis, trismus, infection, hemorrhage, dentoalveolar fracture and dislocation of the neighboring teeth); less permanent (periodontal damage, damage to adjacent teeth, temporomandibular joint damages). Other risks include paresthesia, infection of vital organs, fracture of the jaw and the maxillary tuberosity.

In our case, the indication for surgery was more than evident, since the patient complained about periodic inflammation associated with paraesthesia and a significant impact on his quality of life. The literature indicates that symptomatic pericoronitis can have adverse outcomes, compromising the quality of life and inflicting pain on patients (18), while removal of the third molar positively influences the quality of life outcomes in those with minor symptoms of pericoronitis (19).

The surgical approach using the piezoelectric device has provided more security to avoid further damage to the inferior alveolar nerve. Not surprisingly, the use of piezosurgery for removal of retained wisdom teeth has been promoted with the objective to overcome the limits of the manual or rotary instruments in bone surgery. The cutting action of the piezoelectric element is the result of micro-linear ultrasonic vibrations. The amplitude of only $20-60 \mu \mathrm{m}$ in the longitudinal direction enables the control of the surgical field in all anatomical situations, cutting with extreme precision without damaging the soft tissues accidentally touched, and without generating an excess of temperature at the cutting edges.

The potential applications of piezosurgery as a golden standard in maxillofacial surgery are diverse. The versatility of the instrument (due to inserts specifically designed for different purposes) is a further advantage that enables its use both in extraction surgery and in implant surgery. The so-called micrometric cutting allows the removal of internal bone lesions with extreme precision, while avoiding excessive destruction of bone tissue due to invasive approaches. Various manufacturers have designed kit inserts for each specific application, that increase the speed of piezosurgical procedures (20).

In addition, the cavitation effect of the piezoelectric instruments helps in reducing the osseous bleeding and maintains a clean bone surface, while promoting effective cooling to avoid the risks of overheating the bone tissue, when compared with conventional rotary instruments. Piezoelectric surgery provides a precise, less aggressive osteotomy compared with conventional rotatory techniques. However, it has not been found to significantly reduce perioperative pain and anxiety (21).

The simple extraction of third mandibular molars can sometimes result in neurological impairment of lingual and inferior alveolar branches of the trigeminal nerve (22). The incidence varies, as reported in the literature: The lingual nerve $(0.6-8 \%)$ (23); the lower alveolar nerve (0.4-5\%) (24).

Several factors can increase the incidence of complications that can increase the duration and difficulty of the surgery: The age of the patient (because of the completed root formation), the reduction of the periodontal space, the higher density and bone mineralization; the depth of the inclusion; the procedures that lead to exposure of the lower alveolar nerve. Results of a study defining the incidence of operative and postoperative morbidity associated with the removal of impacted mandibular third molars in patients of various ages showed that there is a significant increase in surgical morbidity as patients become older (23). Another study on 9,574 patients of a wide range of ages who had had 16,127 third molars removed concluded that removal of mandibular third molar teeth during the teenage years resulted in decreased operative and postoperative morbidity (24).

Careful preoperative evaluation and proper planning using cone beam computed tomography (CBCT) imaging are indispensable to minimize the risks of intra-operative and postoperative complications.

The difficulty of extraction (including both osteotomy and odontotomy techniques) increases in the presence of deep-situated anatomically elements with an unfavorable morphology, and with the lack of experience of the operator $(25,26)$. In the present case, the contiguity with the dental element represented the highest risk for nerve injury. In surgery of the lower third molar, the possibility of nerve injuries appears more frequently with paraesthesia (abnormal 


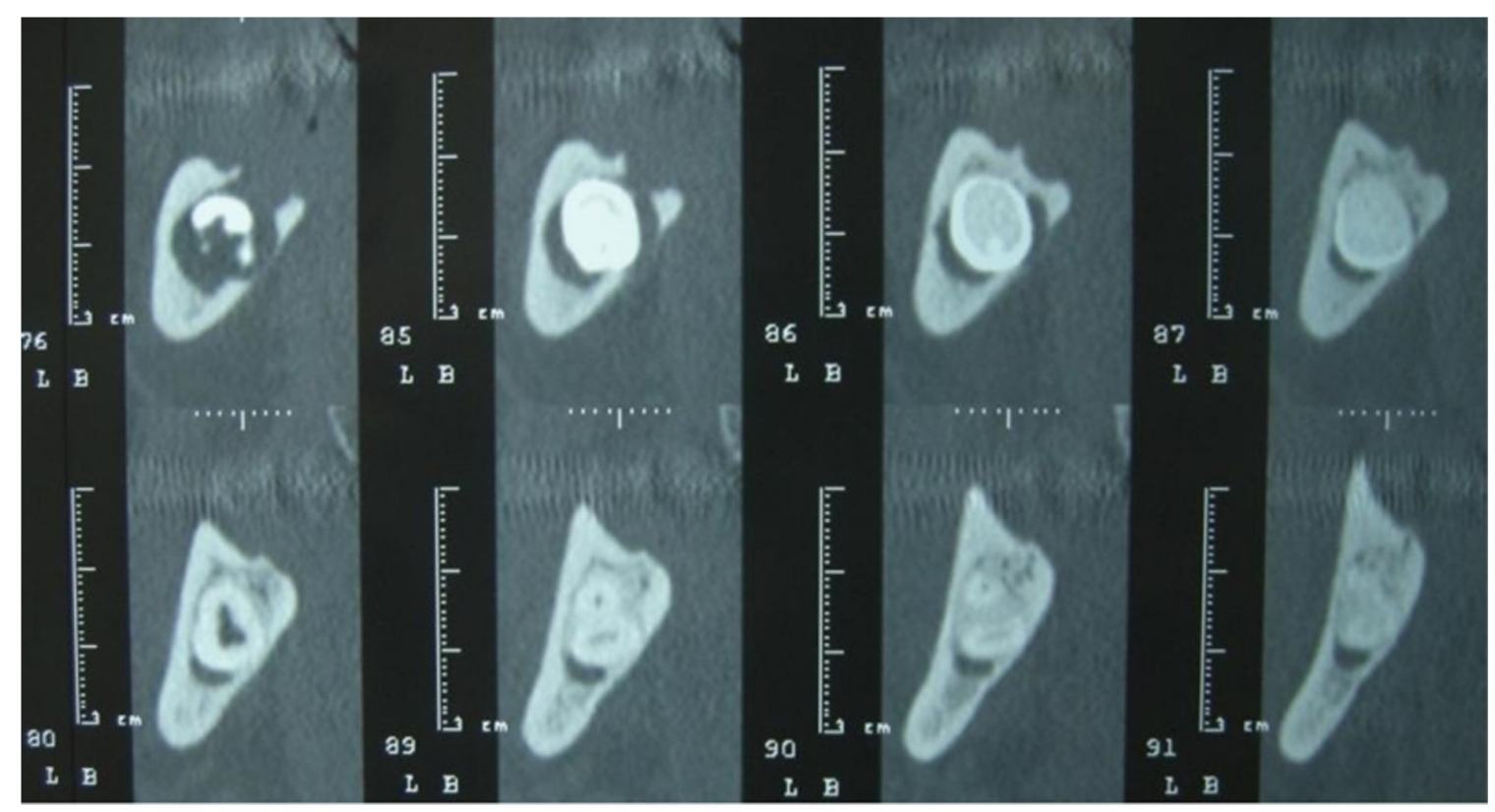

Figure 3. Axial computed tomography (CT) serial sections of the retained tooth. On subsequent sections, note the fact that the mesial aspect of the tooth (both crown and root) forms the roof of the mandibular canal, up to the apex. Furthermore, on next subsequent sections in distal direction, the canal appears to be individualized.

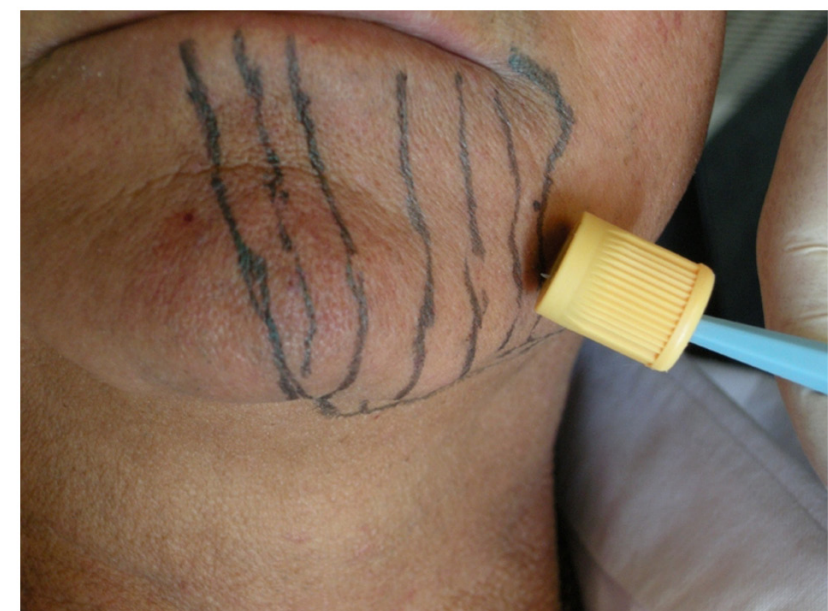

Figure 4. The area of paraesthesia before surgery was delineated with dermatographic pencil following the use of a sharp tip, protected by a plastic cap. The superficial punctures were performed along vertical corridors marked on the skin.

sensitivity) or dysesthesia (abnormal sensitivity associated with burning pain). Similar to our case, canal deviation and interruption of white lines were associated with loss of canal cortication on CBCT in a very recent study, indicating the sensory consequences of a direct contact between the roots and the mandibular canal which required further assessment prior to extraction (27). Contrary to another recent study (28), our case report showed good reliability of radiographic signs seen on OPG on predicting the proximity of the third mandibular root with the mandibular canal, when related to CBCT findings. These complications are considered profoundly serious both because of the medical and psychological sequalae, not to mention the possible legal consequences.

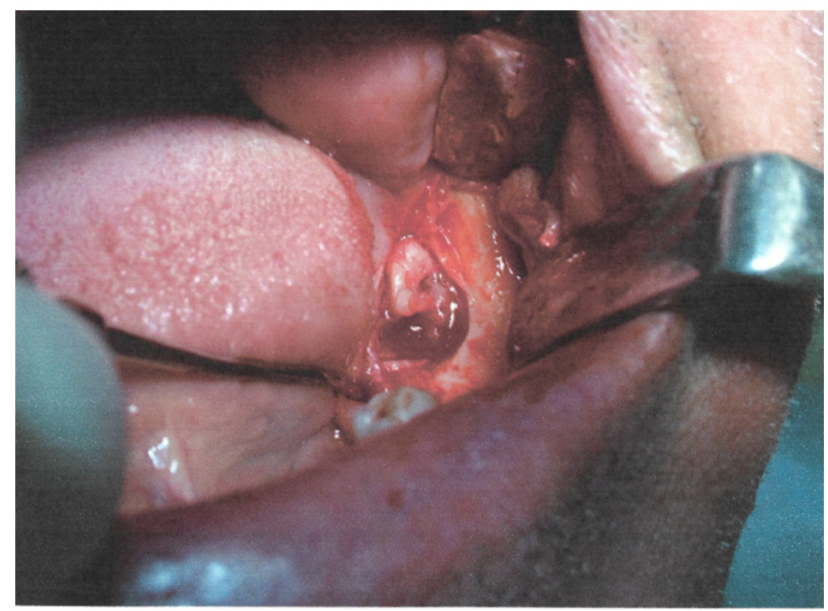

Figure 5. The impacted molar exposed after partial removal of the cortical lid covering the crown. Note the exposed follicular sac folded over the distal aspect of the crown.

The post-operative evolution was characterized by limited swelling of the cheek, the absence of immediate or delayed bleeding, while the pain seemed well controlled with proper medication. After one week, the quality of the wound healing allowed for removal of the sutures, while the hypoaesthetic area was already reduced when compared with the preoperative situation, with considerable subjective benefits for the patient.

In conclusion, hemi-lip paraesthesia is a rare symptom associated preoperatively with dysodontiasis, which suggests the necessity of extraction of the retained causal tooth. The surgical intervention performed with piezoelectric instruments can be considered when there is a need to prevent damage to important structures such as the lower mandibular nerve; 


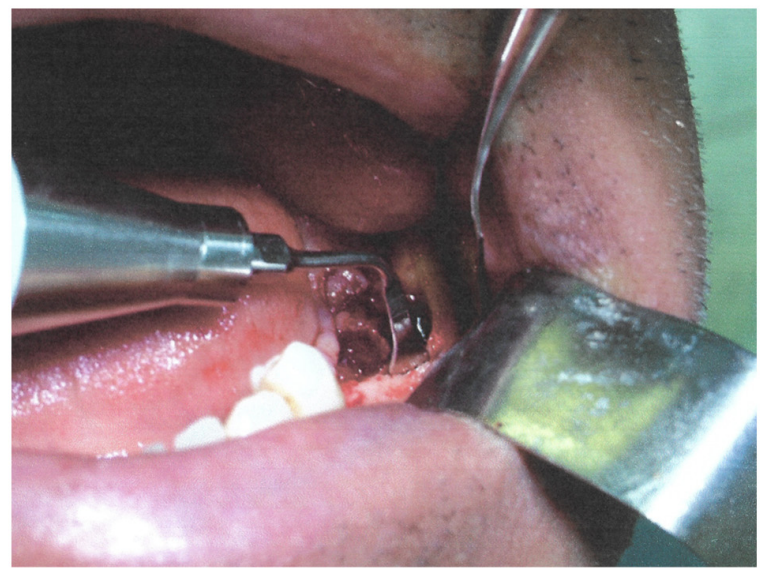

Figure 6. Osteotomy to enlarge the surgical access to the impacted molar crown using piezosurgery. Note the ease of removal of the cortical bone facilitated by the piezosurgical tip.

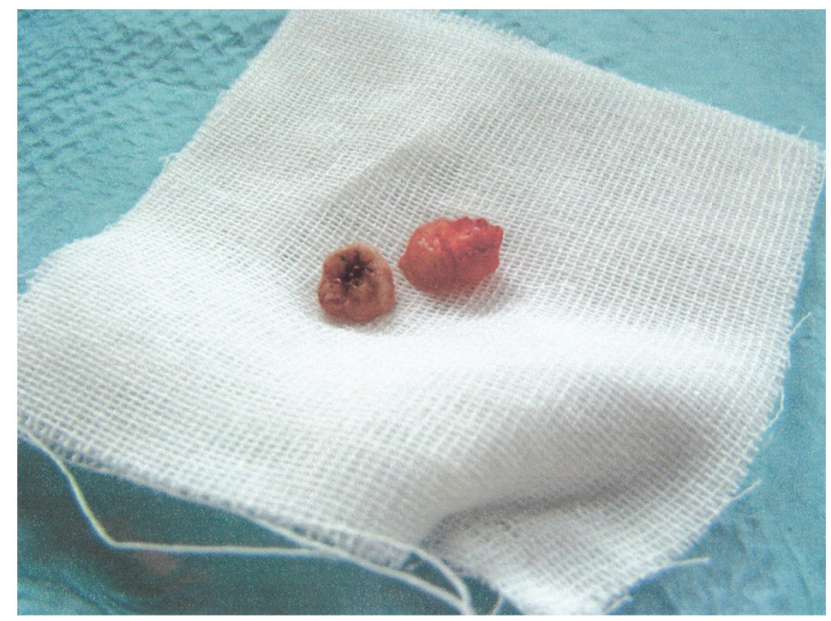

Figure 7. The two fragments of the removed impacted molar, resulted from a single mid-section that separated the crown from the roots in buccal-lingual direction. Note the carious lesion on the occlusal aspect of the molar.

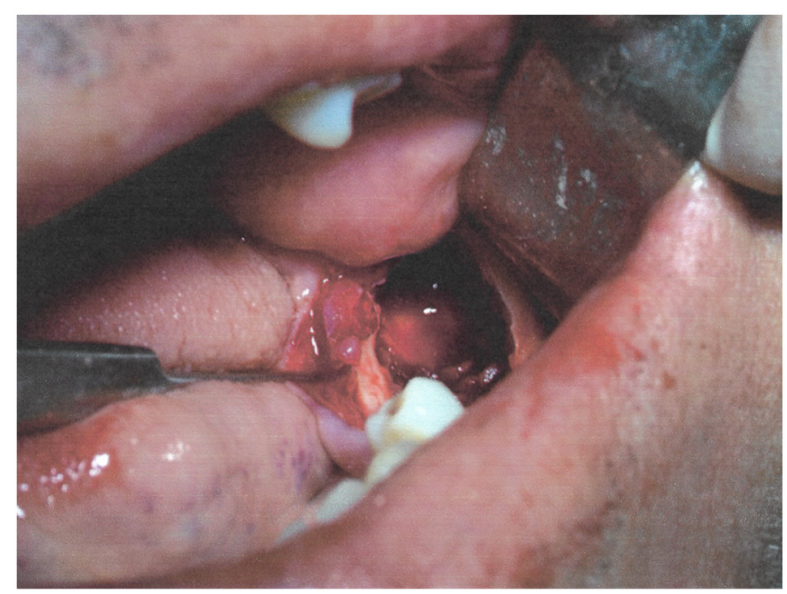

Figure 8 . The residual bony cavity after removal of the impacted molar.

a procedure that, in turn, may support the regression of a contingent paraesthesia.

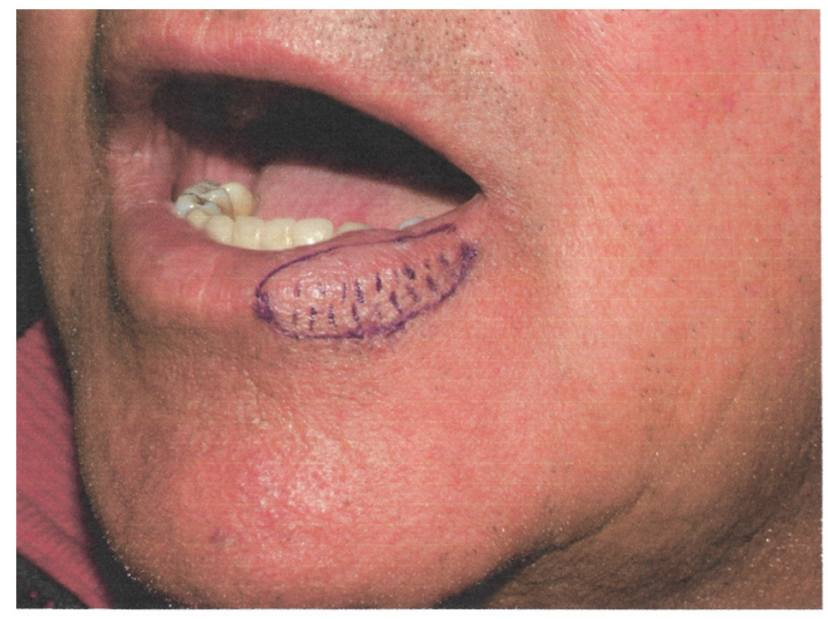

Figure 9. The residual area of paraesthesia at 1-month post-surgery, as delineated with dermatographic pencil. Note the paresthetic area restricted to the distal portion of the lower left hemi-lip.

\section{Acknowledgements}

Not applicable.

\section{Funding}

No funding was received.

\section{Availability of data and materials}

The datasets used/analyzed in this study are available from the corresponding author, upon reasonable request.

\section{Authors' contributions}

MB, SIS, DR contributed substantially to the protocol design. AEdL, FS and SP acquired the preoperatory and intraoperatory clinical data. RG, AR, PS and SS participated in the post-surgical data acquisition and interpretation and were involved in drafting the manuscript and revising it critically for important intellectual content. All authors read and approved the final manuscript.

\section{Ethics approval and consent to participate}

This case report was approved by the Ethics Committee of the Federico II University (no. 345/19/19.02.2020 available on request).

\section{Patient consent for publication}

A written informed consent was obtained from the patient for publication of this case report and any accompanying images. A copy of the written consent is available for review upon request of the Editor-in-Chief of this journal.

\section{Competing interests}

The authors declare that they have no competing interests. 


\section{References}

1. Adeyemo WL, James O, Ogunlewe MO, Ladeinde AL, Taiwo OA and Olojede ACO: Indications for extraction of third molars: A review of 1763 cases. Niger Postgrad Med J 15: 42-46, 2008.

2. Björk A, Jensen E and Palling M: Mandibular growth and third molar impaction. Acta Odontol Scand 14: 231-272, 1956.

3. Valletta G, Bucci E and Matarasso S: Odontostomatology (2 Vol.), (Italian). Piccin, Padova, 1997.

4. NIH consensus development conference for removal of third molars. J Oral Surg 38: 235-236, 1980.

5. Girod SC, Gerlach KI and Krueger G: Cysts associated with long-standing impacted third molars. Int J Oral Maxillofac Surg 22: 110-112, 1993.

6. Regezi JA, Kerr DA and Courtrex RM: Odontogenic tumors: Analysis of 706 cases. J Oral Surg 36: 771-778, 1978.

7. Guven O, Keskin A and Akal K: The incidence of cysts and tumors around impacted third molars. Int J Oral Maxillofac Surg 29: 131-135, 2000

8. Fuster Torres MA, Gargallo Albiol J, Berini Aytés L and Gay Escoda C: Evaluation of the indication for surgical extraction of third molars according to the oral surgeon and the primary care dentist. Experience in the master of oral surgery and implantology at barcelona university dental school. Med Oral Patol Oral Cir Bucal 13: E499-E504, 2008.

9. Knutsson K, Brehmer B, Lysell L and Rohlin M: Pathoses associated with mandibular third molars subjected to removal Oral Surg Oral Med Oral Pathol Oral Radiol Endod 82: 10-17, 1996.

10. Doğan N, Orhan K, Günaydin Y, Köymen R, Okçu K and Uçok O: Unerupted mandibular third molars: Symptoms, associated pathologies, and indications for removal in a Turkish population. Quintessence Int 38: e497-e505, 2007.

11. Punwutikorn J, Waikakul A and Ochareon P: Symptoms of unerupted mandibular third molars. Oral Surg Oral Med Oral Pathol Oral Radiol Endod 87: 305-310, 1999.

12. Barrett AP and Buckley DJ: Selective anesthesias of peripheral branches of the trigeminal nerve due to odontogenic infection. Oral Surg Oral Med Oral Pathol 62: 226-228, 1986.

13. Huang CK, Lui MT and Cheng DH: Use of panoramic radiography to predict postsurgical sensory impairment following extraction of impacted mandibular third molars. J Chin Med Assoc 78: 617-622, 2015.

14. Su N, van Wijk A, Berkhout E, Sanderink G, De Lange J, Wang H and van der Heijden GJMG: Predictive value of panoramic radiography for injury of inferior alveolar nerve after mandibular third molar surgery. J Oral Maxillofac Surg 75: 663-679, 2017.

15. Pell GJ and Gregory B: Impacted mandibular third molars: Classification and modified technique for removal. Dent Dig 39: $330-338,1933$.
16. Maiorana C, Grossi GB, Borgonovo A and Scarpelli M: Surgical extraction of mandibular third molars. Edizioni Sinergie, Milano, pp1-137, 2006 (In Italian).

17. Mercier P and Precious D: Risks and benefits of removal of impacted third molars. A critical review of the literature. J Oral Maxillofac Surg 21: 17-27, 1992.

18. McNutt M, Partrick M, Shugars DA, Phillips C and White RP Jr: Impact of symptomatic pericoronitis on health-related quality of life. J Oral Maxillofac Surg 66: 2482-2487, 2008.

19. Bradshaw S, Faulk J, Blakey GH, Phillips C, Phero JA and White RP Jr: Quality of life outcomes after third molar removal in subjects with minor symptoms of pericoronitis. J Oral Maxillofac Surg 70: 2494-2500, 2012

20. Vercellotti T: Piezosurgery: Essentials in Piezosurgery. Clinical Advantages in Dentistry. Quintessece Publishing, UK, 2009 (In Italian).

21. Topcu SIT, Palancioglu A, Yaltirik M and Koray M: Piezoelectric surgery versus conventional osteotomy in impacted lower third molar extraction: Evaluation of perioperative anxiety, pain, and paresthesia. J Oral Maxillofac Surg 77: 471-477, 2019.

22. Montagna F, Baldoni M and Piras V: Atlas of forensic dentistry. Elsevier Masson, 2005 (In Italian).

23. Bruce RA, Frederickson GC and Small GS: Age of patients and morbidity associated with mandibular third molar surgery. J Am Dent Assoc 101: 240-245, 1980.

24. Osborn TP, Frederickson G Jr, Small IA and Torgerson TS: A prospective study of complications related to mandibular third molar surgery. J Oral Maxillofac Surg 43: 767-769, 1985.

25. Sedaghatfar M, August MA and Dodson TB: Panoramic radiographic findings as predictors of inferior alveolar nerve exposure following third molar extraction. J Oral Maxillofac Surg 63: 3-7, 2005

26. Susarla SM and Dodson TB: Risk factors for third molar extraction difficulty. J Oral Maxillofac Surg 62: 1363-1371, 2004.

27. Al Ali S and Jaber M: Correlation of panoramic high-risk markers with the cone beam CT findings in the preoperative assessment of the mandibular third molars. J Dent Sci 15: 75-83, 2020.

28. Saha N, Kedarnath NS and Singh M: Orthopantomography and Cone-beam computed tomography for the relation of inferior alveolar nerve to the impacted mandibular third molars. Ann Maxillofac Surg 9: 4-9, 2019.

This work is licensed under a Creative Commons Attribution-NonCommercial-NoDerivatives 4.0 International (CC BY-NC-ND 4.0) License. 